\title{
Fabrication of Highly Filled Composites with an Innovative Miniaturized Spouted Bed
}

\author{
Hannah Sophia Rothberg ${ }^{1, * \mathbb{C}}$, Swantje Pietsch ${ }^{1}$, Gerold A. Schneider ${ }^{2}$ and Stefan Heinrich ${ }^{1}$ \\ 1 Institute of Solids Process Engineering and Particle Technology, Hamburg University of Technology, \\ Denickestraße 15, 21073 Hamburg, Germany; swantje.pietsch@tuhh.de (S.P.); stefan.heinrich@tuhh.de (S.H.) \\ 2 Institute of Advanced Ceramics, Hamburg University of Technology, Denickestraße 15, 21073 Hamburg, \\ Germany; g.schneider@tuhh.de \\ * Correspondence: hannah.rothberg@tuhh.de
}

Received: 6 April 2020; Accepted: 24 April 2020; Published: 28 April 2020

\begin{abstract}
In nature bio-composites such as nacre show remarkable mechanical properties due to their complex hierarchical structure and high-volume fraction of its hard component. These composites are highly interesting for structural applications in different branches of industries for mechanical engineering and process technology. The aim of this work was to provide a scalable method for the production of highly filled composites by mimicking the structure of bio-composites. Therefore, composites from iron oxide $\left(\mathrm{Fe}_{2} \mathrm{O}_{3}\right)$ and $\mathrm{SBC}$-polymer (styrene-butadiene block copolymer) were fabricated by using a miniaturized spouted bed with an innovative fluidization gap design. Small iron oxide particles $(25-45 \mu \mathrm{m})$ were fluidized in the spouted bed and coated with a polymer solution via a bottom spray nozzle. Afterwards the coated granules were hot-pressed and the mechanical properties of the obtained composites were tested. By this method composites with a bending strength of up to $6 \mathrm{MPa}$ were fabricated. Although the mechanical properties of these artificial composites are still lower than those of the natural role models, it was shown that the spouted bed is a suitable technique for the fabrication of highly filled composites. For further optimization of the mechanical properties more complex and tailor-made starting materials will be used in following studies.
\end{abstract}

Keywords: miniaturized spouted bed; particle coating; highly filled composites; three-point bending test; mechanical properties of composites

\section{Introduction}

Composites are materials which consist of two or more components, where one phase is the continuous matrix and the other phase is embedded discontinuously [1]. Natural composite materials like bones, teeth, and nacre show remarkable mechanical properties due to their complex hierarchical and graded structure and high-filling degrees of the hard constituent.

Natural hard tissues are usually composites consisting of hard ceramic particles such as aragonite embedded in a soft polymer matrix. The mechanical properties of the composite often exceed those of the single components. For example, nacre consists of 95 vol. \% of its hard constituent aragonite, which is embedded in a biopolymeric matrix as presented in Figure 1a. As single material aragonite is very fragile but in combination with a small amount of ductile biopolymer the strength and toughness are increased 20-30 times [2]. There are more examples of natural composites which show those remarkable mechanical properties like bone or teeth, in which the hard constituent is hydroxyapatite.

For structural applications there is a high interest in applying nature's design principles to manufacture synthetic composites as commercial materials. By utilization of customized components those composites could be used for various structural applications. Many different methods were reported for the production of bio-inspired composites. For example, Finnemore et al. [3] developed a route of 
layer-by-layer assembly of organic films on $\mathrm{CaCO}_{3}$. Other attempts were done by Bonderer et al. [4] using a combination of gel-casting and hot-pressing or biomineralization by Oaki and Imai [5].

Although a lot of progress has been made, there are still challenges in the development of a fast and scalable process to obtain thin organic films in dense, highly filled composites. Within the scope of the SFB 986 project, where this current work is associated with too, Brandt et al. [6] and Wolff et al. [7] developed a process route for the fabrication of multi-level hierarchical composites by a combination of emulsion polymerization and spouted bed coating. They received first promising results regarding the structure and the mechanical properties with composites from $\mathrm{TiO}_{2}$ and PMMA/PVB (poly-(methyl methacrylate)/poly vinyl butyral) (see Figure 1b). Eichner et al. [8] as well worked on the fabrication of highly filled composites from fine copper particles and polymer by the spouted bed process. The authors received an optimum for mechanical properties defined as bending strength and Young's modulus with regard to the copper volume ratio. They further enhanced the mechanical properties by an increased aspect ratio of the copper particles which was achieved by milling the granules after the spouted bed process [9].



(a)

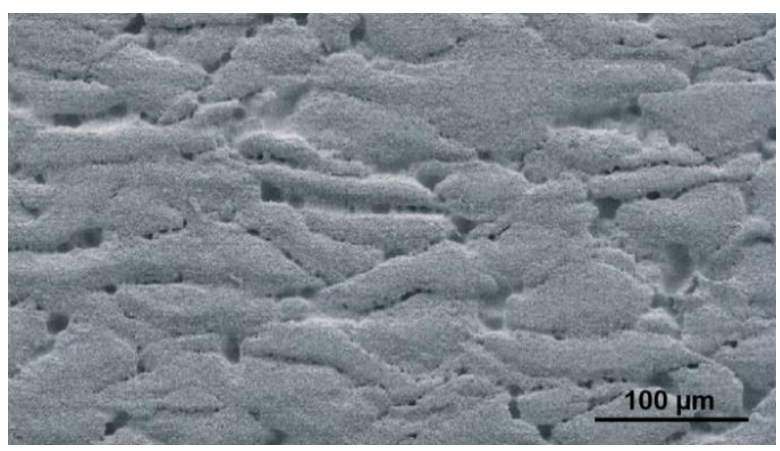

(b)

Figure 1. (a) Schematic of the structure of nacre with aragonite tablets and polymeric film after Espinosa et al. [2]; (b) SEM-image of the composites from $\mathrm{TiO}_{2}$ with a PMMA/PVB (poly-(methyl methacrylate)/poly vinyl butyral) film produced by Brandt et al. [6].

This work was conducted within a research project with the aim of the production of hierarchical, self-similar materials (SFB 986 — “Tailor-made, multi-scale materials systems"). In this project composites are to be made from tailor-made super-crystalline iron oxides and high-performance polymers, and their properties should resemble natural role models as nacre. As the tailor-made starting materials are very expensive, preliminary tests with commercial iron oxide particles and polymer were carried out to investigate the influence of the iron oxide content on the composites on the mechanical properties. The results of these preliminary tests are presented in this work. For the composite production, fine iron oxide particles were used. It is known that the coating of particles smaller than $100 \mu \mathrm{m}$ is challenging due to the agglomeration and adhesion behavior based on their high cohesivity [10]. For this reason, a spouted bed process was used to coat the iron oxide particles with a polymeric film. The spouted bed is a special form of the fluidized bed with the difference that the gas is supplied via a narrow gap instead of a porous distributor plate. As a result, higher shear forces and fluidization velocities are achieved and thus very fine, cohesive and as well very coarse and heavy particles can be fluidized. Due to the high gas inlet velocities and the conical geometry of the expansion zone, a circulating, mushroom-shaped particle flow is achieved. For this work a miniaturized spouted bed was developed to enable the usage of organic solvents for tailor-made polymers and small amounts of bed material.

During the experiment, the particles are fluidized by the fluidization gas and wetted with a polymer solution. As the particles rise, the solvent evaporates and the polymer remains on the particles. Due to the conical geometry of the expansion zone, the velocity of the particles decreases and they fall back into the annulus of the spouted bed. This creates a circulating movement of the particles, which 
leads to repeated contacts with the polymer solution. It is known that the transport processes, such as heat, mass, and momentum transfer, are much more intensive in spouted beds in comparison to classical fluidized beds [11]. This is beneficial when using a polymer solution for coating, where the vaporization of the solvent is critical for the coating step.

\section{Materials and Methods}

For the hard component in the composites iron oxide particles $\left(\mathrm{Fe}_{2} \mathrm{O}_{3}\right.$, Harold Scholz and Co. $\mathrm{GmbH}$, Recklinghausen, Germany) with a $\mathrm{d}_{50,3}$ of $33 \mu \mathrm{m}$ were used. $\mathrm{Fe}_{2} \mathrm{O}_{3}$ (hematite) is a very hard material, easy to handle and to source, and commonly used as pigments. In Chicot et al. [12] the mechanical properties of $\alpha-\mathrm{Fe}_{2} \mathrm{O}_{3}$ are investigated and for hematite-aggregates the elastic modulus can vary between 200 and 350 GPa while for powder grain aggregates the mechanical properties are much lower ("from some GPa to a few hundred" [12] p. 864). For the soft component SBC-polymer (styrene-butadiene block copolymer) (Styroflex@) 2G66, BASF SE, Ludwigshafen, Germany) was chosen. This copolymer combines the hard and brittle behavior of styrene with the more ductile properties of butadiene. A good processability, memory effect, and a very high elongation at break are the resulting properties of this copolymer [13]. The tensile modulus/flexural modulus of the SBC-polymer is given as 120-140 MPa by the manufacturer. The tensile/flexural strength is given as $4 \mathrm{MPa}$ [14].

The fabrication and testing of the highly filled composites consisted of three main steps. First the iron oxide particles were coated with the polymer in the spouted bed process. Afterwards the composite granules were compacted to platelets. Bars were cut from the composite platelet and the mechanical properties were investigated by three-point bending test. The experimental procedure is shown schematically in Figure 2.

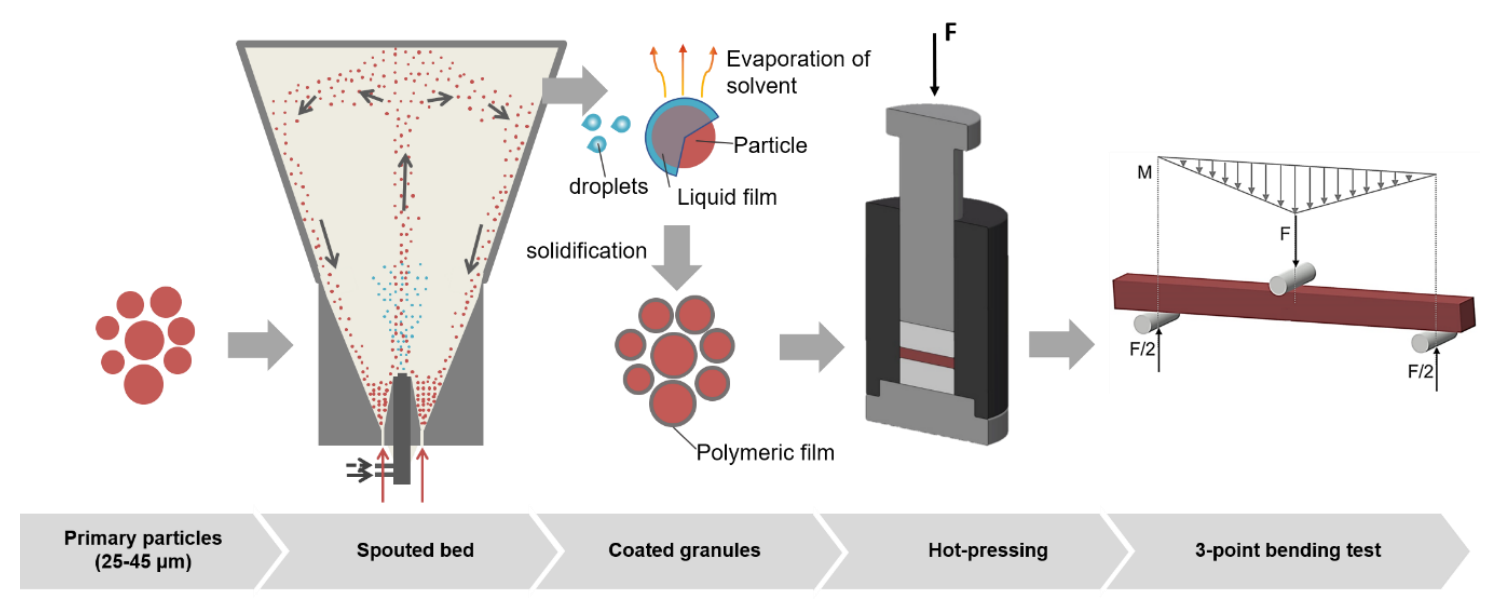

Figure 2. Experimental procedure for the fabrication of highly filled composites by spouted bed spray coating.

Additionally, the polymer content of the granules was determined thermogravimetrically and the granule size was obtained by dynamic image analysis. For the investigation of the coating quality and polymer layer thickness samples were analyzed by SEM (scanning electron microscopy (Supra), Carl Zeiss Microscopy GmbH, Jena, Germany) in combination with the focused ion beam technique (FIB, Helios NanoLab DualBeam, FEI). Furthermore, the composites were analyzed by tomography (Nanotom (C) XCT system, GE Sensing and Inspection Technologies GmbH, Wunstorf, Germany).

\subsection{Spouted Bed Process}

A miniaturized conical-cylindrical spouted bed was used for the coating process of the iron oxide particles with the polymer. The process chamber, the expansion zone, and the lower part of the relaxation zone of the spouted bed are conical and the upper part of the relaxation zone is cylindrical 
(see Figure 3). The fluidization gap of the process chamber is an annular gap with an area of $97.4 \mathrm{~mm}^{2}$ (gap size of $1 \mathrm{~mm}$ ), which was specifically constructed for the fluidization of fine particles and is adjustable in width. By changing the gap width, higher or lower fluidization velocities above the annular gap can be reached and, therefore, the process chamber can be adjusted to material with different fluidization behavior.

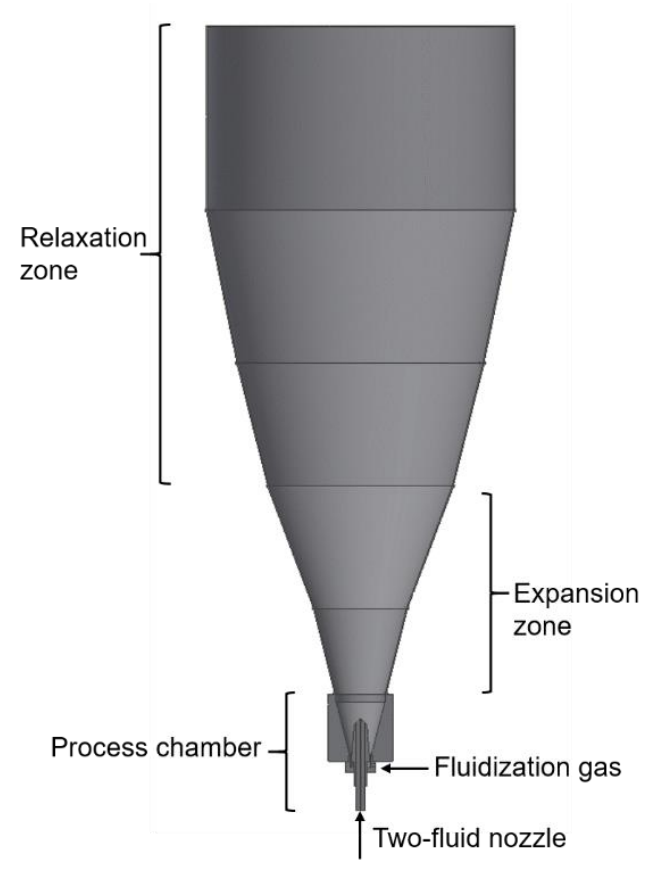

(a)

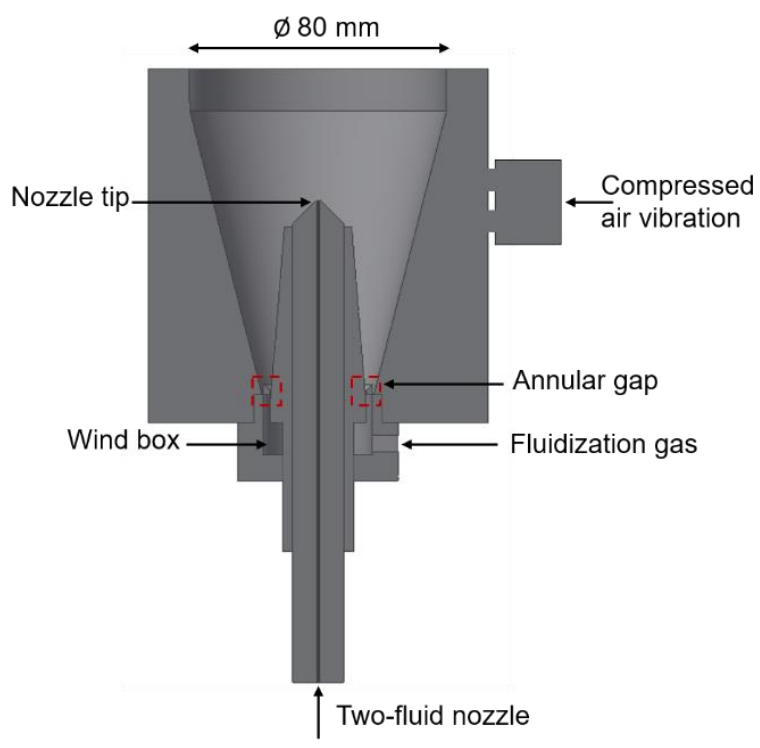

(b)

Figure 3. (a) Cross-section of the miniaturized spouted bed with total height of $1.25 \mathrm{~m}$ used for coating of fine particles; (b) cross-section of the process chamber with annular gap for fluidization gas and two-fluid nozzle for polymer solution (gradient of process chamber wall: $12^{\circ}$ ).

A total of $30 \mathrm{~g}$ of the fine iron oxide particles with a $\mathrm{d}_{50,3}$ of $33 \mu \mathrm{m}$ were introduced into the process chamber and the fluidization was started. Through a two-fluid nozzle (Model 970/3 S1, Düsen-Schlick $\mathrm{GmbH}$, Coburg, Germany), which was installed in bottom-spray configuration, the polymer solution was injected during the experiment. In the experiments the size of the nozzle tip and the configuration of the nozzle wind box were varied (see Table 1). For the polymer solution, the block copolymer SBC (styrene-butadiene block copolymer) was dissolved in THF (Tetrahydrofuran ROTIPURAN, Carl Roth $\mathrm{GmbH}$ and Co. KG, Karlsruhe, Germany). The solution was filled into glass syringes and injected during the experiment via a syringe pump (Legato 270, World Precision Instruments Germany GmbH, Friedberg, Germany) with a specific spaying rate. Nitrogen was used as fluidization and atomization gas to ensure explosion protection. The experiment was started with a lower fluidization volume flow but due to the increasing weight of the particles through granulation and agglomeration, a continuous increase of the volume flow was necessary to guarantee a stable fluidization with the final values mentioned in Table 1. The atomization gas volume flow was kept constant during one experiment. A comparatively high atomizing air flow was necessary to prevent clogging of the nozzle tip. 
Table 1. Process parameter settings for the experiments.

\begin{tabular}{lcccccccc}
\hline \multicolumn{1}{c}{ Process Parameter * } & \multicolumn{7}{c}{ Experiment Number } \\
\hline & 1 & 2 & 3 & 4 & 5 & 6 & 7 & 8 \\
Fluidization gas volume flow (L/min) & 7 & 7 & 7 & 7 & 8 & 5 & 5 & 5 \\
Atomization gas volume flow (L/min) & 9 & 8 & 8 & 8 & 8 & 8 & 8 & 7 \\
Spraying rate (mL/min) & 0.45 & 0.4 & 0.35 & 0.35 & 0.35 & 0.4 & 0.35 & 0.35 \\
Fluidization gap width (mm) & 1 & 1 & 0.8 & 1 & 1 & 1 & 1 & 1 \\
Wind box configuration (nozzle) ** & 2 & 2 & 1 & 1 & 1 & 1 & 1 & 1 \\
Nozzle tip size (mm) & 0.5 & 0.5 & 0.5 & 0.5 & 0.5 & 0.5 & 0.5 & 0.3 \\
Polymer (g) for coating solution & 1.5 & 1.5 & 1.5 & 1.5 & 1.5 & 1.46 & 1.46 & 1.46 \\
THF (g) for coating solution & 100 & 100 & 100 & 100 & 100 & 73 & 73 & 73 \\
\hline
\end{tabular}

* The process parameters for those experiments were adjusted due to experience from previous tests in order to find a stable process control. The aim of this study was not to conduct a parameter study. It was shown that those experiments result in different amounts of polymer in the granules, whose influence on the composite properties is the central object of this study; ** the size of the wind box at the two-fluid nozzle (Model 970/3 S1, Düsen-Schlick $\mathrm{GmbH}$, Germany) can be configurated. A scale from zero to five specifies the configuration of the wind box (zero: closed atomization gap; five: wide open gap) and therefore influence the velocity of the atomization gas.

The spouted bed system is controlled by a PLC (programmable logic controller). The process temperature was $23^{\circ} \mathrm{C}$. The process temperature on the one hand has to be high enough to guarantee a fast evaporation of the solvent to avoid the contact of wet particle with the walls of the spouted bed. On the other hand, the solvent THF must not evaporate too quickly on the nozzle tip, as this would clog the nozzle. Therefore, those parameters need to be optimized for each particle-polymer combination. As the iron oxide particles are quite cohesive due to their shape and particle size distribution, a compressed air vibrator (NCT1, Netter GmbH, Wiesbaden, Germany) was attached to the process chamber to assist the fluidization and avoid particle sticking to the wall.

After the spouted bed process the polymer content was determined by thermogravimetric analysis. Therefore, two samples each were heated to $550{ }^{\circ} \mathrm{C}$ in a muffle furnace for $1 \mathrm{~h}$ in air atmosphere and weighed before and after the heat treatment. The weight difference equals the organic content (polymer) of the granules. Due to possible oxidation of the iron oxide during combustion, there is the possibility of a small deviation in the polymer content. However, since all measurements were carried out in the same way, this deviation is the same across all tests and is therefore initially negligible. This deviation could be avoided in future experiments by a comparable measurement using a TGA under an $\mathrm{N}_{2}$ atmosphere. Due to the changes in process parameters in the spouted bed experiments, different polymer contents were measured. The particle size of the granules was measured by dynamic image analysis (Camsizer ${ }^{\circledR}$ XT, Retsch, Haan, Germany).

\subsection{Compaction and Sawing}

For the production of composite pellets from the granules, a hot press was used (PWV 300, Paul-Otto Weber $\mathrm{GmbH}$, Remshalden, Germany). Therefore, about $15 \mathrm{~g}$ of the granules were filled in a stencil. First, the temperature was set at $80^{\circ} \mathrm{C}$ with a heating rate of $5 \mathrm{~K} / \mathrm{min}$ and a small pressure of $40 \mathrm{MPa}$. After the target temperature was reached, a higher pressure of $500 \mathrm{MPa}$ was set and held for $1 \mathrm{~h}$. Thus, pellets with a diameter of $40 \mathrm{~mm}$ and height of about $4 \mathrm{~mm}$ were produced. The density of the pellet was determined by pycnometer. Then the pellet was attached on a plate with wax for sawing. Thus, 6-12 rectangular bars with a width of approx. $2.5 \mathrm{~mm}$ were cut by a diamond saw (Brilliant 200, ATM GmbH, Mammelzen, Germany). The number of produced bars varied because some of them broke during sawing or separation from the plate.

\subsection{Bending Test}

Bending strength and Young's modulus were determined by a three-point bending test in a highly stiff loading device. Two support rolls with a center-distance of $20 \mathrm{~mm}$ were mounted in a rigid metal frame and the upper support was positioned in the middle. With a constant velocity of $2 \mu \mathrm{m} / \mathrm{s}$ the 
upper support moved downwards resulting in an increasing force to the bending bar. The force and displacement were reported by a LabVIEW program. By this method 6-12 bending bars from each produced composite were tested. An example of a bending curve from the composites bars is shown in Figure 4.

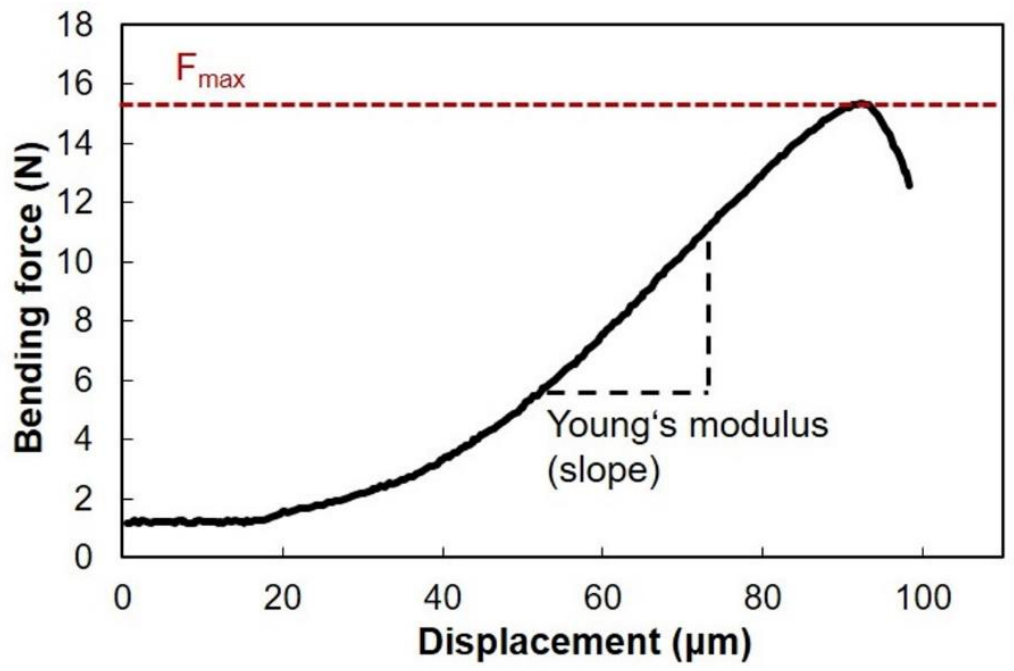

Figure 4. Bending curve observed by three-point bending test of composite bars with 65 vol. $\% \mathrm{Fe}_{2} \mathrm{O}_{3}$. With the $F_{\max }$-value the bending strength and with the slope the Young's modulus is determined.

For the calculation of the bending strength, the following Equation (1) according to DIN EN 843-1 [15] was used:

$$
\sigma_{f}=\frac{3 F_{\max } l}{2 b h^{2}}
$$

with $\sigma_{f}$ for bending strength, $F_{\max }$ for maximum force at breakage point $(\mathrm{N}), l$ for distance between lower supports $(\mathrm{mm}), b$ for width of sample $(\mathrm{mm})$, and $h$ for height of sample $(\mathrm{mm})$.

For the determination of the Young's Modulus the following Equation (2) according to DIN EN 843-2 [16] was used:

$$
E=\frac{\left(F_{2}-F_{1}\right) \cdot l^{3}}{4 \cdot b \cdot h^{3} \cdot\left(d_{2}-d_{1}\right)}
$$

with $E$ for Young's Modulus, $F_{2}$ for upper recorded force $(\mathrm{N}), F_{1}$ for lower recorded force $(\mathrm{N}), d_{2}$ for upper recorded displacement $(\mathrm{m})$, and $d_{1}$ lower recorded displacement $(\mathrm{m})$.

\section{Results and Discussion}

In total eight experiments were realized with the miniaturized spouted bed for this study. Besides the insights regarding the mechanical properties of the composites, the experiments also enabled valuable findings regarding the coating process in the miniaturized spouted bed.

First of all, we were able to show that the miniaturized spouted bed process is able to coat fine iron oxide with varied contents of polymer. It was also shown that several parameters influence the coating process and the resulting polymer/iron oxide content. This includes influenceable process parameters but also some circumstances which were hard to control in our process setup (e.g., changes in the jet stream due to agglomerates adhering to the nozzle tip).

Although some overspray of the polymer solution was observed (30-50 wt.\%) and some of the coated particles stuck to the wall of the reactor (due to increased wall effect in a small-size setup), the spouted bed proved to be a suitable process for the fabrication of composite material. Most of the coated particles (about 60-70 wt.\%) remained in the process chamber after the experiment and even the particles, which stuck to the wall, proved to be mostly coated. 
While many of these findings gave us direction for further experiments regarding the influence of process parameters on the composites, the scope of this study remained on the composition of the composite itself. However, the insights regarding the process parameters will be the base for further studies with the miniaturized spouted bed in order to obtain reproducible results for certain parameter combinations and a regime map for the process.

\subsection{Mechanical Properties and $\mathrm{Fe}_{2} \mathrm{O}_{3}$-Content}

The thermogravimetric measurements showed that composite granules with different polymer concentration (from 3 to $5.5 \mathrm{wt} . \%$ ) were produced in different experiments. The bending strength and the Young's modulus were tested for varying iron oxide to polymer ratios. Each data point represents one experiment where the corresponding polymer content is the arithmetic mean of three thermogravimetric measurements each. The volumetric iron oxide content was calculated via the porosity and the content of the polymer in the composites (see Table S1). The results of the measured bending strength and Young's modulus are shown in Figure 5. The detailed data with standard deviation is given in Table S2 in the Supplementary Material.

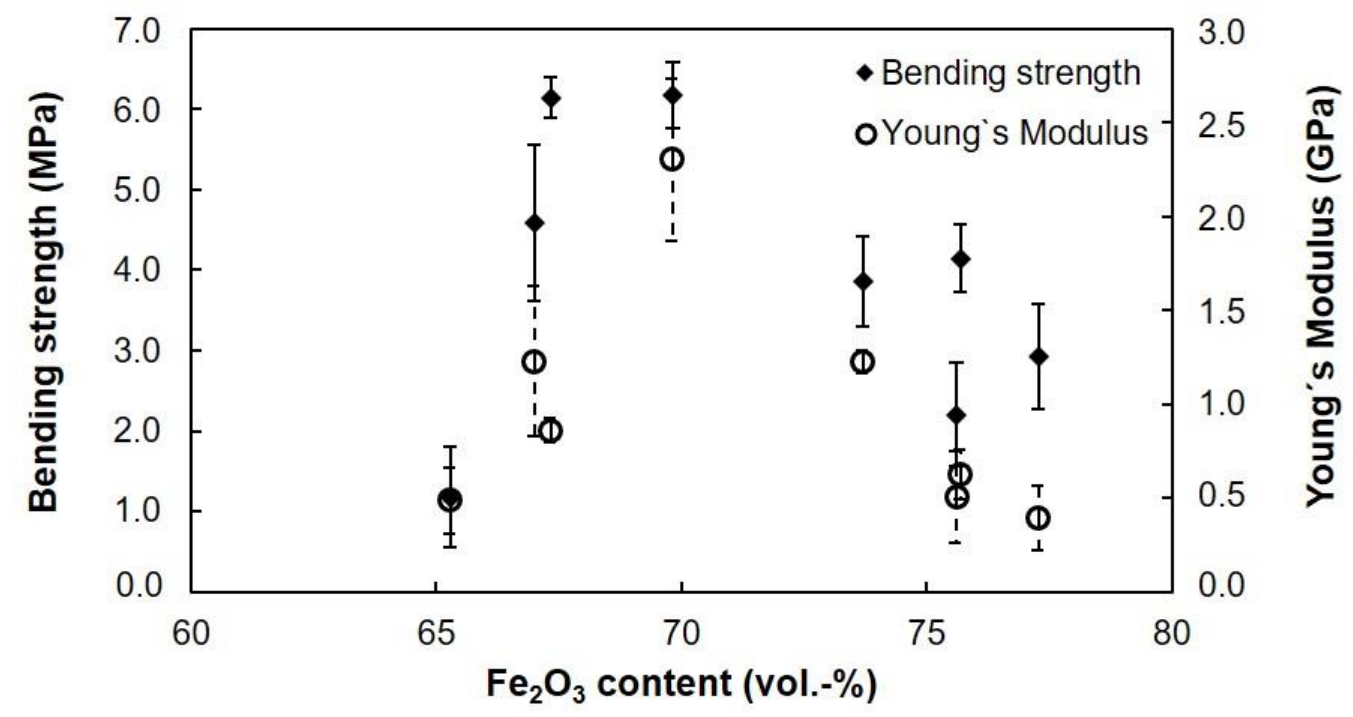

Figure 5. Bending strength and Young's modulus of iron oxide-polymer composites with different iron oxide $\left(\mathrm{Fe}_{2} \mathrm{O}_{3}\right)$ content. Each point shows the arithmetic mean of the mechanical properties for the 6-12 composite bars with a specific $\mathrm{Fe}_{2} \mathrm{O}_{3}$ content.

Figure 5 shows that there is an optimum for bending strength and Young's modulus at about $70 \mathrm{vol} . \%$ iron oxide content. The strength of the produced iron oxide-polymer composites first increases with increasing iron oxide content. This fits to the already mentioned phenomenon of increasing strength in natural composites like nacre due to a thin polymeric film between the elements of the hard constituents [2]. In addition, due to the binder function of the polymeric film it is assumed that the polymer works as a filler of the cavities between the more spherical iron-oxide polymers. Compared to nacre the aragonite has the form of platelets, which form a "brick and mortar" structure, which is additionally advantageous for the mechanical properties. The reason for the decreasing strength with further increasing iron oxide content (and decreasing polymer content) above $70 \mathrm{vol} . \%$ of iron oxide can be explained by the filler-function of the polymer. At a high iron oxide content, the polymer concentration is not high enough to fill all the void space and cavities between the iron oxide particles. For the Young's modulus the same trend is observed. Composites with sufficient filler content (polymer) with less voids and cavities have a higher Young's modulus. Voids between the iron oxide particles which are not filled with polymer can form micro cracks during the bending test. These 
micro cracks result in a lower Young's modulus because the material yields faster under mechanical stress. Otherwise, the Young's modulus of the soft polymer predominates in the case of composites with a higher polymer content (SBC-polymer has a Young's modulus of 0.12 GPa [14]).

Although we assume that the shape of the particle is influenced by the compacting step, the porosity of the pellets with high iron oxide content is still 5-25 vol.\%. Therefore, the void space and the particle-particle-contacts in the composites are weak spots when it comes to the bending test. With lower iron oxide content, the polymer concentration is high enough to fill the voids and form a matrix where the iron oxide particles are embedded. There are less weak spots and the bending strength and the Young's modulus are higher. It is assumed that with further decreased iron oxide content the properties of the soft polymer predominate so that the mechanical properties of the composite get weaker. Overall the mechanical properties result from the interaction between iron oxide content, polymer concentration, and porosity of the composite. Further experiments and investigations are planned to clarify this interaction.

Overall, the obtained bending strength and Young's modulus in this work are low in comparison to other composites obtained from the spouted bed process (copper-PVB composites with $\sigma_{f}=80 \mathrm{MPa}$, $E=25 \mathrm{GPa}$ ) [8]. However, with regard to the mechanical properties of the starting material, especially the copolymer $(E=0.12 \mathrm{GPa})$, it could be shown that the properties were increased in the fabricated composite. With regard to the iron oxide powder it is hard to say if there is already an improvement because there is no fixed value for the Young's modulus in literature for powder grain aggregates ("some GPa to few hundred" [12] p. 864). However, this work shows the results from a preliminary study in preparation for the production of composites from different material systems. Therefore, composites from more valuable functionalized polymers and super-crystalline iron oxide particles should be fabricated to obtain higher strength and higher levels of hierarchical structure.

Aside from the discussed results we are aware of the possibly important influence of the compaction process on the composite structure and properties. Therefore, the influence of pressure and temperature during compaction on the mechanical properties will be investigated in a following study.

\subsection{Particle Size and Coating Layer}

The particle size analysis of the granules showed a particle growth with increasing polymer content (see Figure 6). While the $\mathrm{d}_{50,3}$ of the uncoated $\mathrm{Fe}_{2} \mathrm{O}_{3}$ particles is $33.2 \mu \mathrm{m}$, with 3.8 wt. \% polymer the $\mathrm{d}_{50,3}$ is increased to 39.9 and $46.0 \mu \mathrm{m}$ for $4.8 \mathrm{wt}$.\% polymer content. This agrees with the expectation of particle growth in the spouted bed process due to the additional coating layer on the particle surface. Furthermore, the investigation showed that not only particle growth happens during spouting but there is particle agglomeration as well. This can be shown in Figure $6 \mathrm{~b}$ where the number and size of the peaks at higher particle sizes increases with increasing polymer content for two selected samples with a medium and high polymer content (with regard to the produced composites).

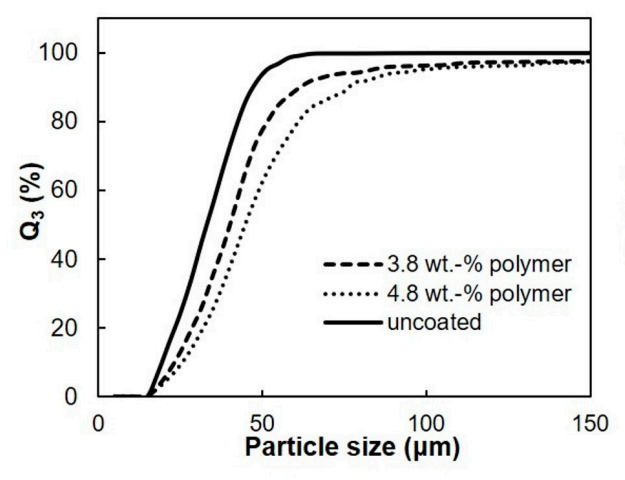

(a)

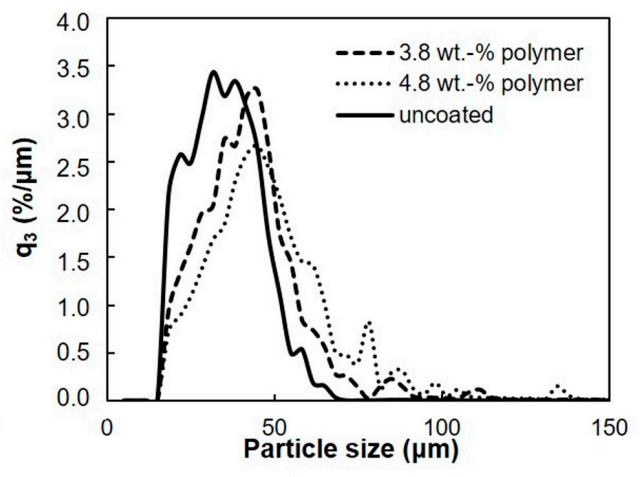

(b)

Figure 6. (a) Cumulative particle size distribution and (b) density distribution of the uncoated $\mathrm{Fe}_{2} \mathrm{O}_{3}$ particles, granules with $3.8 \mathrm{wt} . \%$ and $4.8 \mathrm{wt} . \%$ polymer. 
The coated samples were analyzed by SEM and FIB. In Figure 7a a cut edge of a pressed composite bar is shown. In contrast to Figure $1 \mathrm{~b}$ no clear differentiation between particle and polymer matrix is possible. This is probably because of the porous inner structure, irregular shape, and therefore good deformability of the iron oxide particles. Due to the very high pressure and temperature applied during the compacting process the particles deform and the polymer spreads in the remaining void spaces. Thus, the pressed composite looks like bulk material under the electron microscope. Other methods like EDX (energy-dispersive X-ray spectroscopy) or tomography (X-ray computed tomography) were carried out to analyze the composite structure. In Figure $7 \mathrm{~b}$ a tomography image is shown. The iron oxide is visible as light grey structure. In between the iron oxide many small white spots can be observed. The white structures are assumed as polymer. Next to the very small spot, some bigger aggregates with size up to $45 \mu \mathrm{m}$ can be seen. It is suspected that those aggregates are polymer which accumulates either during the spouting or during the compression step. Unfortunately, neither the SEM nor the tomography images were able to visualize the inner structure of the composite (polymer matrix-particle-arrangement). For further studies dying the polymer for analysis purpose e.g., with fluorescent dye, would be an option for the visualization of the composite structure.

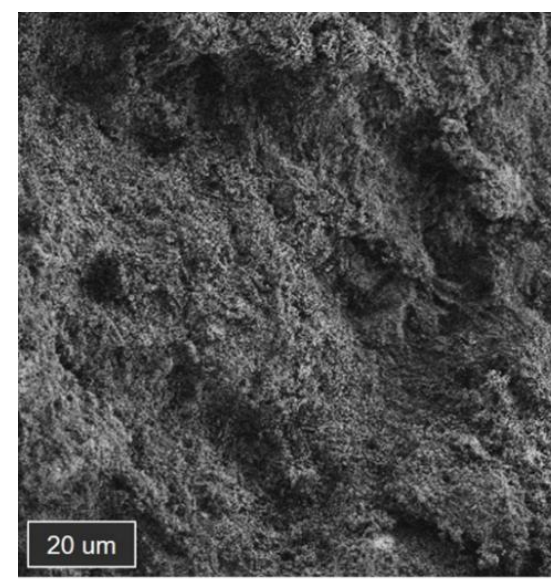

(a)

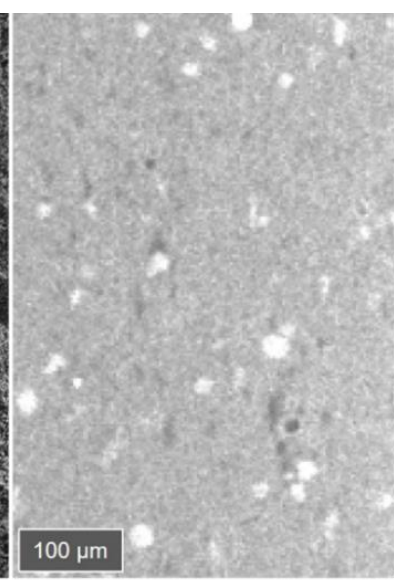

(b)

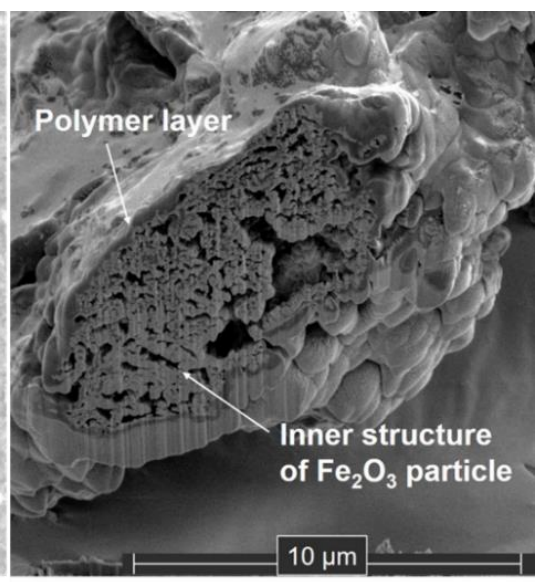

(c)

Figure 7. (a) SEM image of cut edge of composite bar; (b) tomography image of a composite pellet; (c) SEM image of coated $\mathrm{Fe}_{2} \mathrm{O}_{3}$ particle cut by focused ion beam (FIB).

In Figure 7c a SEM image of a coated $\mathrm{Fe}_{2} \mathrm{O}_{3}$ particle is shown. The previously mentioned porous inner structure and irregular shape of the particles can be observed. At this point it is important to note that the high inner porosity of the commercial iron oxide is probably also responsible for the partially high porosity of the composites. By FIB a section of the particle is removed for the analysis of the coating layer. This method for the analysis of a coating layer via cross-sectioning of particles by FIB was reported by Goslinska et al. [17]. In contrast to the porous structure of the iron oxide a denser polymer layer on the particle surface is observed. It can be observed that a good coating of the particles was achieved, however, the layer thickness is likely inhomogeneous.

Overall it could be shown that the particles were successfully coated with the miniaturized spouted bed. An objective for further studies will be the optimization of the coating layer with regard to homogeneity due to the high importance of a uniform polymer matrix in the composite.

\section{Conclusions}

This work shows the influence of the iron oxide $\left(\mathrm{Fe}_{2} \mathrm{O}_{3}\right)$ content on the mechanical properties (bending strength and Young's modulus) of iron oxide-polymer composites. The iron oxide-polymer composites consist of iron oxide particles coated with a polymeric film in a spouted bed process. Therefore, a process chamber with an annular fluidization gap was constructed for the fluidization of 
fine hematite particles $\left(\mathrm{d}_{50}=33 \mu \mathrm{m}\right)$. After the coating and compacting step, the mechanical properties were determined by three-point-bending test. The results show an optimum of 70 vol. $\% \mathrm{Fe}_{2} \mathrm{O}_{3}$ with a bending strength of about $6 \mathrm{MPa}$ and a Young's modulus of about $2.5 \mathrm{GPa}$. The existence of an optimum results from the interaction of iron oxide- and polymer-content and porosity of the compacted composite material.

With regard to the coating quality of the particles further investigations and process optimization are necessary to obtain a homogeneous composite structure. In further studies, a focus will also be placed on improving the mechanical properties of the produced composites. Therefore, other starting material as functionalized particles from supercrystalline iron oxide (magnetite) nanoparticles with a lower inner porosity are considered to produce stronger composite materials. In addition, it is planned to use functionalized polymers (e.g., styrene-butadiene copolymers (S-S/B-S) with introduced hydrogen bonding motifs along the chains from Wittenberg et al. in [18]) which can be crosslinked above a specific temperature. This can lead to a stronger bond between the composite particles and thus improve the mechanical properties. Both the production of the particles, as well as the synthesis of the polymer, are also part of the SFB 86 project.

Supplementary Materials: The following are available online at http://www.mdpi.com/2227-9717/8/5/521/s1, Table S1: Composition analysis of fabricated composite materials, Table S2: Bending strength and Young's modulus of fabricated composite materials (standard deviation included).

Author Contributions: Conceptualization, H.S.R. and S.P.; methodology, H.R.; validation, H.R.; formal analysis, H.S.R.; investigation, H.S.R.; resources, S.H. and G.A.S.; data curation, H.S.R.; writing-original draft preparation, H.S.R.; writing-review and editing, H.S.R., S.P., S.H. and G.A.S.; visualization, H.S.R.; supervision, S.P. and S.H.; project administration, S.H.; funding acquisition, S.H. and G.A.S. All authors have read and agreed to the published version of the manuscript.

Funding: This research was funded by the Deutsche Forschungsgemeinschaft (DFG, German Research Foundation)-project number 192346071-SFB 986.

Conflicts of Interest: The authors declare no conflict of interest.

\section{References}

1. Schulte, K.; Fiedler, B. Structure and Properties of Composite Materials, 2nd ed.; TuTech Innovation: Hamburg, Germany, 2005.

2. Barthelat, F.; Li, C.-M.; Comi, C.; Espinosa, H.D. Mechanical properties of nacre constituents and their impact on mechanical performance. J. Mater. Res. 2006, 21, 1977-1986. [CrossRef]

3. Finnemore, A.; Cunha, P.; Shean, T.; Vignolini, S.; Guldin, S.; Oyen, M.; Steiner, U. Biomimetic layer-by-layer assembly of artificial nacre. Nat. Commun. 2012, 3, 966. [CrossRef] [PubMed]

4. Bonderer, L.J.; Feldmann, K.; Gaukler, L. Platelet-reinforced polymer matrix composites by combined gel-casting and hot-pressing. Part I: Polypropylene matrix composites. Comp. Sci. Technol. 2010, 70, 1958-1965. [CrossRef]

5. Oaki, Y.; Imai, H. The hierarchical architecture and its mimetic material. Angew. Chem. Int. Ed. 2005, 44, 6571-6575. [CrossRef] [PubMed]

6. Brandt, K.; Wolff, M.F.H.; Salikov, V.; Heinrich, S.; Schneider, G.A. A novel method for a multi-level hierarchical composite with brick- and- mortal structure. Sci. Rep. 2013, 3, 2322. [CrossRef] [PubMed]

7. Wolff, M.F.H.; Salikov, V.; Antonyuk, S.; Heinrich, S.; Schneider, G.A. Novel, highly-filled ceramic-polymer composites synthesized by a spouted bed spray granulation process. Compo. Sci. Technol. 2014, 90, 154-159. [CrossRef]

8. Eichner, E.; Salikov, V.; Bassen, P.; Heinrich, S.; Schneider, G.A. Using dilute spouting for fabrication of highly filled metal-polymer composite material. Powder Technol. 2017, 316, 426-433. [CrossRef]

9. Eichner, E.; Heinrich, S.; Schneider, G.A. Influence of the particle shape and size on mechanical properties in copper-polymer composites. Powder Technol. 2018, 339, 39-45. [CrossRef]

10. Jono, K.; Ichikawa, H.; Miyamoto, M. Fukumori A review of particulate design for pharmaceutical powders and their production by spouted bed coating. Powder Technol. 2000, 113, 269-277. [CrossRef] 
11. Epstein, N.; Grace, J.R. Spouted and Spout-Fluid Beds: Fundamentals and Applications; Cambridge University Press: Cambridge, UK, 2010.

12. Chicot, D.; Mendoza, J.; Zaoui, A.; Louis, G.; Lepingle, V.; Roudet, F.; Lesage, J. Mechanical properties of magnetite $\left(\mathrm{Fe}_{3} \mathrm{O}_{4}\right)$, hematite $\left(\alpha-\mathrm{Fe}_{2} \mathrm{O}_{3}\right)$ and geothite $(\alpha-\mathrm{FeOOH})$ by instrumented indentation and molecular dynamic analysis. Mater. Chem. Phys. 2011, 129, 862-870. [CrossRef]

13. Elsner, P.; Eyerer, P.; Hirth, T. Kunstoffe, Eigenschaften und Anwendungen, 8th ed.; Springer: Berlin/Heidelberg, Germany, 2012; pp. 401-412.

14. Ineos Styrolution. Technical Datasheet of Styroflex@ 2G66, Styrene-Butadiene Copolymer (SBC); Ineos Styrolution: Frankfurt, Germany, 2016.

15. DIN Deutsches Institut für Normung, e.V. Advanced Technical Ceramics-Mechanical Properties of Monolithic Ceramics at Room Temperature-Part 1: Determination of Flexural Strength (DIN EN 843-1); DIN Deutsches Institut für Normung e.V.: Berlin, Germany, 2008.

16. DIN Deutsches Institut für Normung, e.V. Advanced Technical Ceramics-Mechanical Properties of Monolithic Ceramics at Room Temperature-Part 2: Determination of Young's Modulus, Shear Modulus and Poisson's Ratio (DIN EN 843-2); DIN Deutsches Institut für Normung e.V.: Berlin, Germany, 2007.

17. Goslinska, M.; Selmer, I.; Kleemann, C.; Kulozik, U.; Smirnova, I.; Heinrich, S. Novel technique for measurement of coating layer thickness of fine and porous particles using focused ion beam. Particuology 2019, 42, 190-198. [CrossRef]

18. Wittenberg, E.; Meyer, A.; Eggers, S.; Abetz, V. Hydrogen bonding and thermoplastic elastomers-A nice couple with temperature-adjustable mechanical properties. Soft Matter 2018, 14, 2701-2711. [CrossRef] [PubMed]

(C) 2020 by the authors. Licensee MDPI, Basel, Switzerland. This article is an open access article distributed under the terms and conditions of the Creative Commons Attribution (CC BY) license (http://creativecommons.org/licenses/by/4.0/). 